\title{
Combination of Two Powerful Methods for Solving Nonlinear Partial Differential Equations
}

\section{Djelloul Ziane $^{1}$ and Mountassir Hamdi Cherif ${ }^{2}$}

Laboratory of Mathematics and its Applications (LAMAP), University of Oran1 Ahmed Ben Bella, Oran, 31000, Algeria

e-mail: ${ }^{1}$ djeloulz@yahoo.com, ${ }^{2}$ mountassir27@yahoo.fr

\begin{abstract}
The idea of this work is to combine the homotopy analysis method with a new transform called "Shehu transform". The goal is to try to take advantage of this transform to give a better speed to the homotopy analysis method to solve nonlinear partial differential equations. Several examples are given to re-confirm the efficiency of the suggested algorithm.
\end{abstract}

\section{Introduction}

Integral transformations are defined by integrals, which play an important role in solving linear ordinary differential equations or linear partial differential equations. Among the most important of these integral transformations are the Laplace transform and the Fourier transform, that appeared in the 18th century. In addition, several transformations have appeared during the last 30 years, such as, Sumudu transform [25], natural transform [14], Ezaki transform [6], Aboodh transform [1], ZZ-transform [32] and the last transformation appeared is the Shehu transform [14], which is developed this year.

In order to take advantage of these transformations and to use them to solve

Received: October 25, 2019; Accepted: December 22, 2019

2010 Mathematics Subject Classification: 35A08, 44A05, 35G25, 65H20.

Keywords and phrases: homotopy analysis method, Shehu transform, nonlinear partial differential equations.

Copyright () 2020 Djelloul Ziane and Mountassir Hamdi Cherif. This is an open access article distributed under the Creative Commons Attribution License, which permits unrestricted use, distribution, and reproduction in any medium, provided the original work is properly cited. 
nonlinear differential equations, the researchers are using them a lot now, and they have combined them with some usual methods, such as, the homotopy analysis method (HAM). This method was developed in 1992 by Liao Shijun in his PhD dissertation ([15], [16], [17], [18]) and was used by many researchers to solve linear and nonlinear differential equations ([2], [3], [4], [10], [21]).

Among the work done by combining this method with some transforms, we will mention some of them, the homotopy analysis method coupled with Laplace transform ([8], [9], [12], [19], [24]), homotopy analysis Sumudu transform method ([7], [22], [23]), homotopy Natural transform method ([5], [13], [29]), homotopy analysis Elzaki transform method ([27], [28]) and homotopy analysis Aboodh transform method [30].

The basic idea of this paper consists of combining two important methods for solving nonlinear differential equations. The first method is homotopy analysis method, and the second is a new transform developed recently [14]. The combination of these two methods gives us an efficient method to solve nonlinear partial differential equations.

The present paper has been organized as follows: In Section 2 some basic definitions and properties of the Shehu transform, in addition, two propositions concerning the partial derivative. In Section 3 an analysis of the proposed method. In Section 4 the application of this method on three suggested examples. Finally, the conclusion follows.

\section{Basics of Shehu Transform}

In this section, we will provide the basic definition of Shehu transform, basic theories and some important properties [20], and two propositions concerning the general formula of the Shehu transform of partial derivatives.

\subsection{Definition of transformation and existence theorem}

Definition 2.1. The Shehu transform of the function $v(\tau)$ of exponential order is defined over the set of functions,

$$
A=\left\{v(\tau): \exists N, k_{1}, k_{2}>0,|v(\tau)|<N \exp \left(\frac{|\tau|}{k_{i}}\right), \text { if } \tau \in(-1)^{j} \times[0, \infty)\right\}
$$

by the following integral

$$
\hat{S}[v(\tau)]=[V(s, u)]=\int_{0}^{\infty} \exp \left(\frac{-s \tau}{u}\right) v(\tau) d \tau
$$




$$
=\lim _{\alpha \rightarrow \infty} \int_{0}^{\alpha} \exp \left(\frac{-s \tau}{u}\right) v(\tau) d \tau, s>0, u>0 .
$$

It converges if the limit of the integral exists, and diverges if not.

The inverse Shehu transform is given by

$$
\hat{S}^{-1}[V(s, u)]=v(\tau), \text { for } \tau \geq 0 .
$$

Equivalently

$$
v(\tau)=\hat{S}^{-1}[V(s, u)]=\frac{1}{2 \pi i} \int_{\alpha-i \infty}^{\alpha+i \infty} \frac{1}{u} \exp \left(\frac{s \tau}{u}\right) V(s, u) d s,
$$

where $s$ and $u$ are the Shehu transform variables, and $\alpha$ is a real constant and the integral in (2.4) is taken along $s=\alpha$ in the complex plane $s=x+i y$.

Theorem 2.1. The sufficient condition for the existence of Shehu transform. If the function $v(\tau)$ is piecewise continuing in every finite interval $0 \leq \tau \leq \beta$ and of exponential order $\alpha$ for $\tau>\beta$. Then its Shehu transform $V(s ; u)$ exists.

Proof. See [20].

\subsection{Derivative of Shehu transform}

Theorem 2.2. Derivative of Shehu transform. If the function $v^{(n)}(t)$ is the $n$-th derivative of the function $v(\tau) \in A$, then its Shehu transform is defined by

$$
\hat{S}\left[v^{(n)}(\tau)\right]=\frac{s^{n}}{u^{n}} V(s, u)-\sum_{n=0}^{\infty}\left(\frac{s}{u}\right)^{n-(k+1)} v^{k}(0) .
$$

When $n=1,2$, and 3 in (2.5) above, we obtain the following derivatives with respect to $t$.

$$
\begin{gathered}
\hat{S}\left[v^{\prime}(\tau)\right]=\frac{s}{u} V(s, u)-v(0), \\
\hat{S}\left[v^{\prime \prime}(\tau)\right]=\frac{s^{2}}{u^{2}} V(s, u)-\frac{s}{u} v(0)-v^{\prime}(0),
\end{gathered}
$$




$$
\hat{S}\left[v^{\prime \prime \prime}(\tau)\right]=\frac{s^{3}}{u^{3}} V(s, u)-\frac{s^{2}}{u^{2}} v(0)-\frac{s}{u} v^{\prime}(0)-v^{\prime \prime}(0) .
$$

Proof. See [20].

2.3. Some properties. Below, we summarize some important properties of this transform, and their proof can be found in [20].

1. (Linearity): $\hat{S}[(\alpha f(\tau))+\beta g(\tau)]=\alpha \hat{S}[f(\tau)]+\alpha \hat{S}[g(\tau)]$.

2. (Change of scale). $\hat{S}[f(\beta \tau)]=\frac{u}{\beta} V\left(\frac{s}{\beta}, u\right)$.

In the following table, we will present other properties of this transformation:

\begin{tabular}{|c|c|l|c|}
\hline$v(\tau)$ & $\hat{S}[v(\tau)]$ & $v(\tau)$ & $\hat{S}[v(\tau)]$ \\
\hline 1 & $\frac{u}{s}$ & $\sin a \tau$ & $\frac{a u^{2}}{s^{2}+a^{2} u^{2}}$ \\
\hline$\tau$ & $\frac{u^{2}}{s^{2}}$ & $\cos a \tau$ & $\frac{u s}{s^{2}+a^{2} u^{2}}$ \\
\hline$\frac{\tau^{n}}{n !}, n=0,1,2, .$. & $\left(\frac{u}{s}\right)^{n+1}$ & $\sinh a \tau$ & $\frac{a u^{2}}{s^{2}-a^{2} u^{2}}$ \\
\hline$\frac{\tau^{n} \exp (a \tau)}{n !}$ & $\frac{u^{n+1}}{(s-a u)^{n+1}}$ & $\cosh a \tau$ & $\frac{u s}{s^{2}-a^{2} u^{2}}$ \\
\hline
\end{tabular}

Proposition 2.3. If $\frac{\partial v(r, \tau)}{\partial \tau}$ and $\frac{\partial^{2} v(r, \tau)}{\partial \tau^{2}}$ exist, and by using integration by parts, we obtain

$$
\begin{gathered}
\hat{S}\left[\frac{\partial v(r, \tau)}{\partial \tau}\right]=\frac{s}{u} V(r, s, u)-v(r, 0), \\
\hat{S}\left[\frac{\partial^{2} v(r, \tau)}{\partial \tau^{2}}\right]=\frac{s^{2}}{u^{2}} V(r, s, u)-\frac{s}{u} v(r, 0)-\frac{\partial v(r, 0)}{\partial \tau} .
\end{gathered}
$$

Proof. The use of integration by parts, gives

$$
\begin{aligned}
\hat{S}\left[\frac{\partial v(r, \tau)}{\partial \tau}\right] & =\int_{0}^{\infty} e^{\frac{-s t}{u}} \frac{\partial v(r, \tau)}{\partial \tau} d t=\lim _{r \rightarrow \infty} \int_{0}^{\tau} e^{\frac{-s t}{u}} \frac{\partial v(r, \tau)}{\partial \tau} d \tau \\
& =\lim _{t \rightarrow \infty}\left(\left[v(r, \tau) e^{\frac{-s \tau}{u}}\right]_{0}^{t}+\frac{s}{u} \int_{0}^{t} e^{\frac{-s \tau}{u}} v(r, \tau) d \tau\right) \\
& =\frac{s}{u} V(r, s, u)-v(r, 0) .
\end{aligned}
$$


Let $\frac{\partial v(r, \tau)}{\partial \tau}=w(r, \tau)$, then, by using (2.5) and (2.9), we get

$$
\begin{aligned}
\hat{S}\left[\frac{\partial^{2} v(r, \tau)}{\partial \tau^{2}}\right] & =\hat{S}\left[\frac{\partial w(r, \tau)}{\partial \tau}\right]=\frac{s}{u} \hat{S}[w(r, \tau)]-w(r, 0) \\
& =\frac{s}{u} \hat{S}\left[\frac{\partial v(r, \tau)}{\partial \tau}\right]-\frac{\partial v(r, 0)}{\partial \tau} \\
& =\frac{s^{2}}{u^{2}} V(r, s, u)-\frac{s}{u} v(r, 0)-\frac{\partial v(r, 0)}{\partial \tau}
\end{aligned}
$$

Proposition 2.4. Let $V(r, s, u)$ is the Shehu transform of $v(r, \tau)$. Then one has

$$
\hat{S}\left[\frac{\partial^{n} v(r, \tau)}{\partial \tau^{n}}\right]=\frac{s^{n}}{u^{n}} V(r, s, u)-\sum_{k=0}^{n-1}\left(\frac{s}{u}\right)^{n-(k+1)} \frac{\partial^{k} v(r, 0)}{\partial \tau^{k}} .
$$

Proof. To demonstrate the validity of the formula (2.11), we use mathematical induction.

If $n=1$ and according to the formula (2.11), we obtain

$$
\hat{S}\left[\frac{\partial v(r, \tau)}{\partial \tau}\right]=\frac{s}{u} V(r, s, u)-v(r, 0) .
$$

So, according to (2.9) we note that the formula holds when $n=1$.

Assume inductively that the formula holds for $n$, so that

$$
\hat{S}\left[\frac{\partial^{n} v(r, \tau)}{\partial \tau^{n}}\right]=\frac{s^{n}}{u^{n}} V(r, s, u)-\sum_{k=0}^{n-1}\left(\frac{s}{u}\right)^{n-(k+1)} \frac{\partial^{k} v(r, 0)}{\partial \tau^{k}}
$$

and show that it stays true at rank $n+1$. Let $\frac{\partial^{n} v(x, t)}{\partial t^{n}}=w(x, t)$ and according to (2.9) and (2.13), we have

$$
\hat{S}\left[\frac{\partial^{n+1} v(r, \tau)}{\partial \tau^{n+1}}\right]=\hat{S}\left[\frac{\partial w(r, \tau)}{\partial \tau}\right]=\frac{s}{u} \hat{S}(w(r, \tau))-w(r, 0)
$$




$$
\begin{aligned}
& =\frac{s}{u}\left[\frac{s^{n}}{u^{n}} V(r, s, u)-\sum_{k=0}^{n-1}\left(\frac{s}{u}\right)^{n-(k+1)} \frac{\partial^{k} v(r, 0)}{\partial \tau^{k}}\right]-\frac{\partial^{n} v(r, 0)}{\partial \tau^{n}} \\
& =\frac{s^{n+1}}{u^{n+1}} V(r, s, u)-\sum_{k=0}^{n-1}\left(\frac{s}{u}\right)^{n+1-(k+1)} \frac{\partial^{k} v(r, 0)}{\partial \tau^{k}}-\frac{\partial^{n} v(r, 0)}{\partial \tau^{n}} \\
& =\frac{s^{n+1}}{u^{n+1}} V(r, s, u)-\sum_{k=0}^{n}\left(\frac{s}{u}\right)^{n+1-(k+1)} \frac{\partial^{k} v(r, 0)}{\partial \tau^{k}} .
\end{aligned}
$$

Thus by the principle of mathematical induction, the formula (2.11) holds for all $n \geq 1$.

\section{Homotopy Analysis Shehu Transform Method}

To illustrate the basic idea of this method, we consider a general nonlinear nonhomogeneous partial differential equation:

$$
\frac{\partial^{m} W(r, \tau)}{\partial \tau^{m}}+L(W(r, \tau))+R(W(r, \tau))=h(r, \tau)
$$

where $m=1,2, \ldots$, and $\frac{\partial^{m} W(r, \tau)}{\partial \tau^{m}}$ is the partial derivative of the function $W(r, \tau)$ of order $m, L$ is the linear differential operator, $R$ represents the general nonlinear differential operator, and $h(r, \tau)$ is the source term.

Applying the Shehu transform (denoted in this paper by $\hat{S}$ ) on both sides of (3.1), we get

$$
\hat{S}\left[\frac{\partial^{m} W(r, \tau)}{\partial \tau^{m}}\right]+\hat{S}[L(W(r, \tau))+R(W(r, \tau))-h(r, \tau)]=0 .
$$

Using the property of the Shehu transform, we have the following formula

$$
\begin{aligned}
\hat{S}[W(r, \tau)] & -\sum_{k=0}^{m-1}\left(\frac{u}{s}\right)^{k+1} \frac{\partial^{k} W(x, 0)}{\partial \tau^{k}} \\
& +\frac{u^{m}}{s^{m}} \hat{S}[L(W(r, \tau))+R(W(r, \tau))-h(r, \tau)]=0 .
\end{aligned}
$$


Define the nonlinear operator

$$
\begin{aligned}
N[\phi(r, \tau ; p)]= & \hat{S}[\phi(r, \tau ; p)]-\sum_{k=0}^{m-1}\left(\frac{u}{s}\right)^{k+1} \frac{\partial^{k} \phi(r, 0, p)}{\partial \tau^{k}} \\
& +\frac{u^{m}}{s^{m}} \hat{S}[L \phi(r, \tau ; p)+R \phi(r, \tau ; p)-h(r, \tau ; p)] .
\end{aligned}
$$

By means of homotopy analysis method [15], we construct the so-called the zeroorder deformation equation

$$
(1-p) \hat{S}\left[\phi(r, \tau ; p)+\phi_{0}(r, \tau, 0)\right]=\operatorname{phH}(r, \tau) N[\phi(r, \tau ; p)],
$$

where $p$ is an embedding parameter and $p \in[0,1], H(r, \tau) \neq 0$ is an auxiliary function, $h \neq 0$ is an auxiliary parameter, $\hat{S}$ is an auxiliary linear Shehu transform operator. When $p=0$ and $p=1$, we have

$$
\left\{\begin{array}{l}
\phi(r, \tau ; 0)=W_{0}(r, \tau) \\
\phi(r, \tau ; 1)=W(r, \tau)
\end{array}\right.
$$

When $P$ increases from 0 to 1 , the $\phi(r, \tau, p)$ various from $W_{0}(r, \tau)$ to $W(r, \tau)$. Expanding $\phi(r, \tau ; p)$ in Taylor series with respect to $p$, we have

$$
\phi(r, \tau ; p)=W_{0}(r, \tau)+\sum_{m=1}^{+\infty} W_{m}(r, \tau) p^{m}
$$

where

$$
W_{m}(r, \tau)=\left.\frac{1}{m !} \frac{\partial^{m} \phi(r, \tau ; p)}{\partial p^{m}}\right|_{p=0}
$$

When $p=1$, the (3.7) becomes

$$
W(r, \tau)=W_{0}(r, \tau)+\sum_{m=1}^{+\infty} W_{m}(r, \tau)
$$

Define the vectors

$$
\vec{W}_{n}=\left\{W_{0}(r, \tau), W_{1}(r, \tau), W_{2}(r, \tau), \ldots, W_{n}(r, \tau)\right\} .
$$


Differentiating (3.5) $m$-times with respect to $p$, then setting $p=0$ and finally dividing them by $m$ !, we obtain the so-called $m$ th-order deformation equation

$$
\hat{S}\left[W_{m}(r, \tau)-\chi_{m} W_{m-1}(r, \tau)\right]=h p H(r, \tau) \Re_{m}\left(\vec{W}_{m-1}(r, \tau)\right),
$$

where

$$
\mathfrak{R}_{m}\left(\vec{W}_{m-1}(r, \tau)\right)=\left.\frac{1}{(m-1) !} \frac{\partial^{m-1} N(r, \tau ; p)}{\partial p^{m-1}}\right|_{p=0},
$$

and

$$
\chi_{m}= \begin{cases}0, & m \leq 1 \\ 1, & m>1\end{cases}
$$

Applying the inverse Shehu transform on both sides of (3.11), we can obtain

$$
W_{m}(r, \tau)=\chi_{m} W_{m-1}(r, \tau)+\hat{S}^{-1}\left[h p H(r, \tau) \Re_{m}\left(\vec{W}_{m-1}(r, \tau)\right)\right]
$$

The $m$-th deformation equation (3.13) is a linear which can be easily solved. So, the solution of (3.1) can be written into the following form

$$
W(r, \tau)=\sum_{m=0}^{M} W_{m}(r, \tau)
$$

when $M \rightarrow \infty$, we can obtain an accurate approximation solution of (3.1).

\section{Application of the HASTM}

In this section, we apply the homotopy analysis transform method (HAM) coupled with Shehu transform, to solve some nonlinear partial differential equations.

Example 4.1. First, we consider the following nonlinear KdV equation:

$$
W_{\tau}+W W_{r}-W_{r r}=0
$$

with the initial condition

$$
W(r, 0)=r
$$

In view of the HAM technique and assuming $H(r, \tau)=1$, we construct the so-called the zero-order deformation equation as follows 


$$
(1-p) \hat{S}\left[\phi(r, \tau ; p)-\phi_{0}(r, \tau, 0)\right]=p h N[\phi(r, \tau ; p)],
$$

where

$$
N[\phi(r, \tau, p)]=\hat{S}[\phi(r, \tau ; p)]-\frac{u}{s} r+\frac{u}{s} \hat{S}\left[\phi(r, \tau, p)(\phi(r, \tau, p))_{r}-(\phi(r, \tau, p))_{r r}\right] .
$$

The series solution of (4.1) is given by (3.9). Thus, we obtain the $m$-th order deformation equation:

$$
W_{m}(r, \tau)=\chi_{m} W_{m-1}(r, \tau)+h A^{-1}\left[\Re_{m}\left(\vec{W}_{m-1}(r, \tau)\right)\right]
$$

with

$$
\begin{aligned}
\mathfrak{R}_{m}\left(\vec{W}_{m-1}(r, \tau)\right)= & \hat{S}\left[W_{m-1}(r, \tau)\right]-\frac{u}{s}\left(1-\chi_{m}\right) r \\
& +\frac{u}{s} \hat{S}\left[\sum_{i=0}^{m-1} W_{i}\left(W_{m-1-i}\right)_{r}-\left(W_{m-1}\right)_{r r}\right]
\end{aligned}
$$

and

$$
\chi_{m}= \begin{cases}0, & m \leq 1 \\ 1, & m>1\end{cases}
$$

Let us take the initial approximation as

$$
W_{0}(r, \tau)=r .
$$

According to (4.5) and (4.6), the formulas of the first terms are given by

$$
\begin{aligned}
W_{1}(r, \tau)= & h \hat{S}^{-1}\left(\frac{u}{s} \hat{S}\left[W_{0}\left(W_{0}\right)_{r}-\left(W_{0}\right)_{r r}\right]\right), \\
W_{2}(r, \tau)= & (1+h) W_{1}(r, \tau)+h \hat{S}^{-1}\left(\frac{u}{s} \hat{S}\left[W_{0}\left(W_{1}\right)_{r}+W_{1}\left(W_{0}\right)_{r}-\left(W_{1}\right)_{r r}\right]\right), \\
W_{3}(r, \tau)= & (1+h) W_{2}(r, \tau) \\
& +h \hat{S}^{-1}\left(\frac{u}{s} \hat{S}\left[W_{0}\left(W_{2}\right)_{r}+W_{1}\left(W_{1}\right)_{r}+W_{2}\left(W_{0}\right)_{r}-\left(W_{2}\right)_{r r}\right]\right),
\end{aligned}
$$


Using the initial approximation (4.8) and the iteration formulas (4.9), we obtain

$$
\begin{aligned}
& W_{0}(r, \tau)=r, \\
& W_{1}(r, \tau)=(h) r \tau, \\
& W_{2}(r, \tau)=\left(h+h^{2}\right) r \tau+h^{2} r \tau^{2}, \\
& W_{3}(r, \tau)=(h)(1+h)^{2} r \tau+3\left(h^{2}+h^{3}\right) r \tau^{2}+h^{3} r \tau^{3},
\end{aligned}
$$

The other components of the (HASTM) can be determined in a similar way. Finally, the approximate solution of (4.1) in a series form is given by

$$
\begin{aligned}
W(r, \tau) & =W_{0}(r, \tau)+W_{1}(r, \tau)+W_{2}(r, \tau)+W_{3}(r, \tau)+\cdots \\
& =r+\left[3 h+3 h^{2}+h^{3}\right] r \tau+\left[4 h^{2}+3 h^{3}\right] r \tau^{2}+h^{3} r \tau^{3}+\cdots
\end{aligned}
$$

Substituting $h=-1$ in (4.11), the approximate solution of (4.1), given as follows

$$
W(r, \tau)=r\left[1-\tau+\tau^{2}-\tau^{3}+\cdots\right] .
$$

And in the closed form, is given by

$$
W(r, \tau)=\frac{r}{1+\tau},|\tau|<1 .
$$

This result represents the exact solution of the equation (4.1) as presented in [31].

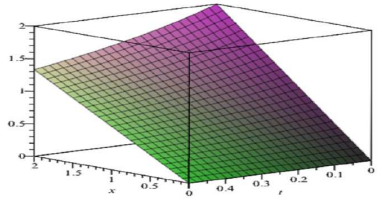

(a)

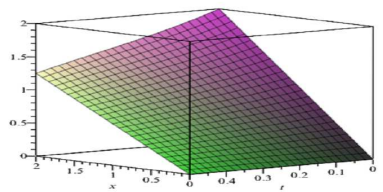

(b)

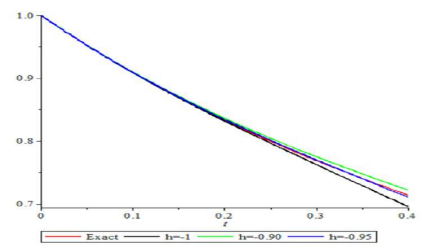

Figure 1. (a) The graph of the exact solution, (b) The graph of the approximate solution for four terms and in the case $h=-1$, (c) The graph of the exact solution and the approximate solution for four terms with different values for $h$ in the case $r=1$. 
Example 4.2. Second, we consider the following nonlinear gas dynamics equation:

$$
W_{\tau}+W W_{r}-W(1-W)=0, \quad \tau>0
$$

with the initial condition

$$
W(r, 0)=e^{-r}
$$

In view of the HAM technique and assuming $H(r, \tau)=1$, we construct the so-called the zero-order deformation equation as follows

$$
(1-p) \hat{S}\left[\phi(r, \tau ; p)-\phi_{0}(r, \tau, 0)\right]=p h N[\phi(r, \tau ; p)],
$$

where

$$
N[\phi(r, \tau, p)]=\hat{S}[\phi(r, \tau ; p)]-\frac{u}{s} e^{-r}+\frac{u}{s} \hat{S}\left[W W_{r}-W(1-W)\right]
$$

The series solution of (4.14) is given by (3.9). Thus, we obtain the $m$-th order deformation equation

$$
W_{m}(r, \tau)=\chi_{m} W_{m-1}(r, \tau)+h \hat{S}^{-1}\left[\Re_{m}\left(\vec{W}_{m-1}(r, \tau)\right)\right]
$$

with

$$
\begin{aligned}
\Re_{m}\left(\vec{W}_{m-1}(r, \tau)\right)= & \hat{S}\left[W_{m-1}(r, \tau)\right]-\frac{u}{s}\left(1-\chi_{m}\right) e^{-r} \\
& +\frac{u}{s} \hat{S}\left[\sum_{i=0}^{m-1} W_{i}\left(W_{m-1-i}\right)_{\chi}+\sum_{i=0}^{m-1} W_{i} W_{m-1-i}-W_{m-1}\right]
\end{aligned}
$$

and

$$
\chi_{m}= \begin{cases}0, & m \leq 1, \\ 1, & m>1\end{cases}
$$

Let us take the initial approximation as

$$
W_{0}(r, \tau)=e^{-r}
$$

According to (4.18) and (4.19), the formulas of the first terms is given by

$$
W_{1}(r, \tau)=h \hat{S}^{-1}\left(\frac{u}{s} \hat{S}\left[W_{0}\left(W_{0}\right)_{r}+\left(W_{0}\right)^{2}-W_{0}\right]\right)
$$




$$
\begin{aligned}
& W_{2}(r, \tau)=(1+h) W_{1}+h \hat{S}^{-1}\left(\frac{u}{s} \hat{S}\left[W_{0}\left(W_{1}\right)_{r}+W_{1}\left(W_{0}\right)_{r}+2 W_{0} W_{1}-W_{1}\right]\right), \\
& W_{3}(r, \tau)=(1+h) W_{2} \\
& +h \hat{S}^{-1}\left(\frac{u}{s} \hat{S}\left[W_{0}\left(W_{2}\right)_{r}+W_{1}\left(W_{1}\right)_{r}+W_{2}\left(W_{0}\right)_{r}+2 W_{0} W_{2}+\left(W_{1}\right)^{2}-W_{2}\right]\right),
\end{aligned}
$$

Using the initial approximation (4.21) and the iteration formulas (4.22), we obtain

$$
\begin{aligned}
& W_{0}(r, \tau)=e^{-r}, \\
& W_{1}(r, \tau)=(-h) e^{-r} \tau, \\
& W_{2}(r, \tau)=(-h)(1+h) e^{-r} \tau+h^{2} e^{-r} \frac{\tau^{2}}{2 !}, \\
& W_{3}(r, \tau)=(-h)(1+h)^{2} e^{-r} \tau+2(1+h) h^{2} e^{-r} \frac{\tau^{2}}{2 !}+\left(-h^{3}\right) e^{-r} \frac{\tau^{3}}{3 !}, \\
& \vdots
\end{aligned}
$$

The other components of the (HASTM) can be determined in a similar way. Finally, the approximate solution of (4.14) in a series form is given by

$$
\begin{aligned}
W(r, \tau)= & W_{0}(r, \tau)+W_{1}(r, \tau)+W_{2}(r, \tau)+W_{3}(r, \tau)+\cdots \\
= & e^{-r}+\left[-3 h-3 h^{2}-h^{3}\right] e^{-r} \tau \\
& +\left[3 h^{2}+2 h^{3}\right] e^{-r} \frac{\tau^{2}}{2 !}+\left(-h^{3}\right) e^{-r} \frac{\tau^{3}}{3 !}+\cdots
\end{aligned}
$$

Substituting $h=-1$ in (4.24), the approximate solution of (4.14), is given as follows

$$
W(r, \tau)=e^{-r}\left[1+\tau+\frac{\tau^{2}}{2 !}+\frac{\tau^{3}}{3 !}+\cdots\right] .
$$

And in the closed form, is given by

$$
W(r, \tau)=e^{-r} e^{\tau}=e^{\tau-r} .
$$


This result (4.26), represents the exact solution of the equation (4.14), as presented in [11].

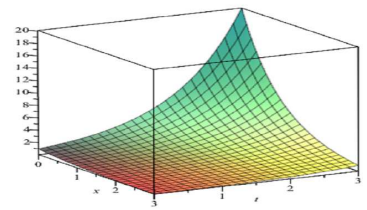

(a)
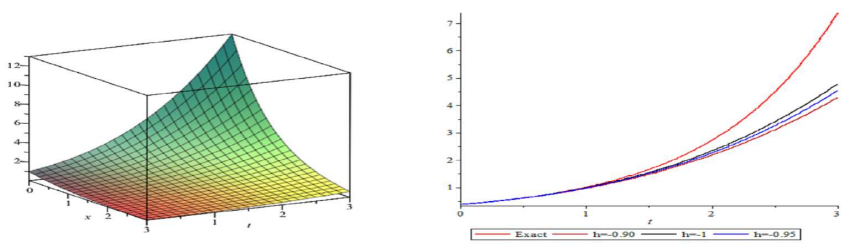

(b)

Figure 2. (a) The graph of the exact solution, (b) The graph of the approximate solution for four terms and in the case $h=-1$, (c) The graph of the exact solution and the approximate solution for four terms with different values for $h$ in the case $r=1$.

Example 4.3. Finally, we consider the following nonlinear partial differential equation:

$$
W_{\tau \tau}-2 \frac{r^{2}}{\tau} W W_{r}=0, \quad \tau>0
$$

with the initial conditions:

$$
W(r, 0)=0, \quad W_{\tau}(r, 0)=r .
$$

In view of the HAM technique and assuming $H(r, \tau)=1$, we construct the so-called the zero-order deformation equation as follows

$$
(1-p) \hat{S}\left[\phi(r, \tau ; p)-\phi_{0}(r, \tau, 0)\right]=p h N[\phi(r, \tau ; p)],
$$

where

$$
N[\phi(r, \tau, p)]=\hat{S}[\phi(r, \tau ; p)]-\frac{u^{2}}{s^{2}} r-\frac{u^{2}}{s^{2}} \hat{S}\left[2 \frac{r^{2}}{\tau} W W_{r}\right] .
$$

The series solution of equation (4.14) is given by (3.9). Thus, we obtain the $m$-th order deformation equation

$$
W_{m}(r, \tau)=\chi_{m} W_{m-1}(r, \tau)+h \hat{S}^{-1}\left[\Re_{m}\left(\vec{W}_{m-1}(r, \tau)\right)\right]
$$


with

$$
\begin{aligned}
\mathfrak{R}_{m}\left(\vec{W}_{m-1}(r, \tau)\right)= & \hat{S}\left[W_{m-1}(r, \tau)\right]-\frac{u^{2}}{s^{2}}\left(1-\chi_{m}\right) r \\
& -\frac{u^{2}}{s^{2}} \hat{S}\left[2 \frac{r^{2}}{\tau} \sum_{i=0}^{m-1} W_{i}\left(W_{m-1-i}\right)_{r}\right],
\end{aligned}
$$

and

$$
\chi_{m}= \begin{cases}0, & m \leq 1 \\ 1, & m>1\end{cases}
$$

Let us take the initial approximation as

$$
W_{0}(r, \tau)=r \tau
$$

According to (4.31) and (4.32), the formulas of the first terms is given by

$$
\begin{aligned}
& W_{1}(r, s, \tau)=-h \hat{S}^{-1}\left(\frac{u^{2}}{s^{2}} \hat{S}\left[2 \frac{r^{2}}{\tau} W_{0}\left(W_{0}\right)_{r}\right]\right), \\
& W_{2}(r, s, \tau)=(1+h) W_{1}-h \hat{S}^{-1}\left(\frac{u^{2}}{s^{2}} \hat{S}\left[2 \frac{r^{2}}{\tau}\left(W_{0}\left(W_{1}\right)_{r}+W_{1}\left(W_{0}\right)_{r}\right)\right]\right), \\
& W_{3}(r, s, \tau)=(1+h) W_{2} \\
&-h \hat{S}^{-1}\left(\frac{u^{2}}{s^{2}} \hat{S}\left[2 \frac{r^{2}}{\tau}\left(W_{0}\left(W_{2}\right)_{r}+W_{1}\left(W_{1}\right)_{r}+W_{2}\left(W_{0}\right)_{r}\right)\right]\right), \\
& \vdots
\end{aligned}
$$

Using (4.34) and the iteration formulas (4.35) we obtain

$$
\begin{aligned}
& W_{0}(x, t)=x t, \\
& W_{1}(x, t)=(-h) \frac{1}{3} x^{3} t^{3}, \\
& W_{2}(x, t)=(1+h)(-h) \frac{1}{3} x^{3} t^{3}+h^{2} \frac{2}{15} x^{5} t^{5},
\end{aligned}
$$




$$
W_{3}(x, t)=(1+h)^{2}(-h) \frac{1}{3} x^{3} t^{3}+(1+h) h^{2} \frac{4}{15} x^{5} t^{5}-h^{3} \frac{17}{315} x^{7} t^{7} .
$$

The other components of the (HASTM) can be determined in a similar way. Finally, the approximate solution of (4.27) in a series form is given by

$$
\begin{aligned}
W(r, \tau)= & r \tau+\left[-3 h-3 h^{2}-h^{3}\right] \frac{1}{3}(r \tau)^{3} \\
& +\left[3 h^{2}+2 h^{3}\right] \frac{2}{15}(r \tau)^{5}+\left(-h^{3}\right) \frac{17}{315}(r \tau)^{7}+\cdots .
\end{aligned}
$$

Substituting $h=-1$ in (4.37), the approximate solution of (4.27), is given as follows

$$
W(r, \tau)=r \tau+\frac{1}{3}(r \tau)^{3}+\frac{2}{15}(r \tau)^{5}+\frac{17}{315}(r \tau)^{7} .
$$

Recall that the exact solution is given by

$$
W(r, \tau)=\tan (r \tau) .
$$

The result (4.39), represents the exact solution of the equation (4.27), as presented in [31].

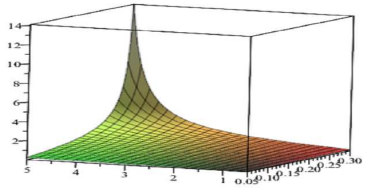

(a)

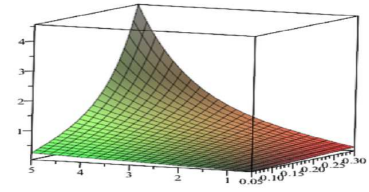

(b)

Figure 3. (a) The graph of the exact solution, (b) The graph of the approximate solution for four terms and in the case $h=-1$, (c) The graph of the exact solution and the approximate solution for four terms with different values for $h$ in the case $r=0.3$.

\section{Conclusion}

On the basis of the results of the suggested examples, the combination of the homotopy analysis method (HAM) with the Shuhu transform, gives us a powerful and efficient method for solving nonlinear partial differential equations. Therefore, it can be 
said that the use of this method to solve other types of nonlinear partial differential equations is certainly possible to achieve the desired results.

\section{References}

[1] K. S. Aboodh, The new integral transform "Aboodh transform", Glob. J. Pure. Appl. Math. 9(1) (2013), 35-43.

[2] S. Abbasbandy, The application of homotopy analysis method to nonlinear equations arising in heat transfer, Phys. Lett. A 360 (2006), 109-113. https://doi.org/10.1016/j.physleta.2006.07.065

[3] M. Ayub, A. Rasheed and T. Hayat, Exact flow of a third grade fluid past a porous plate using homotopy analysis method, Internat. J. Engrg. Sci. 41 (2003), 2091-2103. https://doi.org/10.1016/S0020-7225(03)00207-6

[4] Z. Abbasa, S. Vahdatia, F. Ismaila and A. Karimi Dizicheha, Application of homotopy analysis method for linear integro-differential equations, Int. Math. Forum 5(5) (2010), 237-249.

[5] S. Choudhary, A modified homotopy analysis method to solve fractional telegraph equation, Skit Res. J. 8(1) (2018), 76-79.

[6] T. M. Elzaki and S. M. Elzaki, Applications of new transform "Elzaki transform" to partial differential equations, Glo. J. Pure Appl. Math. 7(1) (2011), 65-70.

[7] V. G. Gupta and P. Kumar, Approximate solutions of fractional biological population model by homotopy analysis Sumudu transform method, Int. J. Sci. Res. 5(5) (2016), 908917. https://doi.org/10.21275/v5i5.NOV163474

[8] V. G. Gupta and S. Gupta, Applications of homotopy analysis transform method for solving various nonlinear equations, W. Appl. Sci. J. 18(12) (2012), 1839-1846.

[9] V. G. Gupta and P. Kumar, Approximate solutions of fractional linear and nonlinear differential equations using Laplace homotopy analysis method, Int. J. Nonlinear Sci. 19(2) (2015), 113-120.

[10] I. Hashim, O. Abdulaziz and S. Momani, Homotopy analysis method for fractional IVPs, Commun. Nonlinear Sci. Numer. Simul. 14 (2009), 674-684. https://doi.org/10.1016/j.cnsns.2007.09.014

[11] H. Jafari, C. Chun, S. Seifi and M. Saeidy, Analytical solution for nonlinear gas dynamic equation by homotopy analysis method, Appl. Appl. Math. 4(1) (2009), 149-154. 
[12] M. M. Khader, S. Kumar and S. Abbasbandy, New homotopy analysis transform method for solving the discontinued problems arising in nanotechnology, Chin. Phys. B. 22(11) (2013), 110201 (1-5). https://doi.org/10.1088/1674-1056/22/11/110201

[13] A. Khan, M. Junaid, I. Khan, F. Ali, K. Shah and D. Khan, Application of homotopy natural transform method to the solution of nonlinear partial differential equations, Sci. Int. (Lahore) 29(1) (2017), 297-303.

[14] Z. H. Khan and W.A. Khan, N-transform properties and applications, NUST J. Eng. Sci. 1 (2008), 127-133.

[15] S. J. Liao, The proposed homotopy analysis technique for the solution of nonlinear problems, Ph.D. Thesis, Shanghai Jiao Tong, University, 1992.

[16] S. J. Liao, Beyond Perturbation: Introduction to Homotopy Analysis Method, Chapman and Hall/CRC Press, Boca Raton, 2003. https://doi.org/10.1201/9780203491164

[17] S. J. Liao, On the homotopy analysis method for nonlinear problems, Appl. Math. Comput. 147 (2004), 499-513. https://doi.org/10.1016/S0096-3003(02)00790-7

[18] S. J. Liao, Notes on the homotopy analysis method: some definitions and theorems, Commun. Nonlinear Sci. Numer. Simul. 14 (2009), 983-997. https://doi.org/10.1016/j.cnsns.2008.04.013

[19] M. S. Mohamed, F. Al-Malki and M. Al-humyani, Homotopy analysis transform method for time-space fractional gas dynamics equation, Gen. Math. Notes 24(1) (2014), 1-16.

[20] S. Maitama and W. Zhao, New integral transform: Shehu transform a generalization of Sumudu and Laplace transform for solving differential equations, Int. J. Anal. Appl. 17(2) (2019), 167-190.

[21] Z. Odibat, S. Momani and H. Xu, A reliable algorithm of homotopy analysis method for solving nonlinear fractional differential equations, Appl. Math. Model. 34 (2010), 593600. https://doi.org/10.1016/j.apm.2009.06.025

[22] R. K. Pandey and H. K. Mishra, Numerical simulation of time-fractional fourth order differential equations via homotopy analysis fractional Sumudu transform method, Ame. J. Num. Anal. 3(3) (2015), 52-64.

[23] S. Rathorea, D. Kumarb, J. Singh and S. Gupta, Homotopy analysis Sumudu transform method for nonlinear equations, Int. J. Industrial Math.. 4(4) (2012), Art. ID IJIM-00204, 13 pp.

[24] K. M. Saad and A.A. Al-Shomrani, An application of homotopy analysis transform method for Riccati differential equation of fractional order, J. Fract. Calc. Appl. 7(1) (2016), 61-72. 
[25] G. K. Watugala, Sumudu transform: a new integral transform to solve differential equations and control engineering problems, Internat. J. Math. Ed. Sci. Tech. 24(1) (1993), 35-43. https://doi.org/10.1080/0020739930240105

[26] A. Wazwaz, The variational iteration method for rational solutions for $\mathrm{KdV}, K(2,2)$, Burgers, and cubic Boussinesq equations, J. Comput. Appl. Math. 207 (2007), 18-23. https://doi.org/10.1016/j.cam.2006.07.010

[27] K. Wang and S. Liu, Application of new iterative transform method and modified fractional homotopy analysis transform method for fractional Fornberg-Whitham equation, J. Nonlinear Sci. Appl. 9 (2016), 2419-2433. https://doi.org/10.22436/jnsa.009.05.42

[28] D. Ziane, Application of homotopy analysis method combined with Elzaki transform for fractional porous medium equation, J. Appr. Theo. Appl. Math. 6 (2016), 1-19.

[29] D. Ziane and M. Hamdi Cherif, Modified homotopy analysis method for nonlinear fractional partial differential equations, Int. J. Anal. Appl. 14(1) (2017), 77-87.

[30] D. Ziane, The combined of homotopy analysis method with new transform for nonlinear partial differential equations, Malaya J. Math. 6(1) (2018), 34-40. https://doi.org/10.26637/MJM0601/0005

[31] D. Ziane and M. Hamdi Cherif, Resolution of nonlinear partial differential equations by Elzaki transform decomposition method, J. Appro. Theo. Appl. Math. 5 (2015), 17-30.

[32] Z. U. Zafar, ZZ transform method, Int. J. Adv. Eng. Glo. Tech. 4(1) (2016), 1605-1611. 